\title{
Registration of Novel Parasiticides under the New 2022 EU Regulation ${ }^{+}$
}

\author{
Sabine Richter, Julia Hunte and Klaus Hellmann*
}

check for

updates

Citation: Richter, S.; Hunte, J.; Hellmann, K. Registration of Novel Parasiticides under the New 2022 EU Regulation. Biol. Life Sci. Forum 2021, 5, 2. https://doi.org/10.3390/ blsf2021005002

Academic Editors: Donato Traversa, Angela Di Cesare and Simone Morelli

Published: 11 October 202

Publisher's Note: MDPI stays neutral with regard to jurisdictional claims in published maps and institutional affiliations.

Copyright: (c) 2021 by the authors. Licensee MDPI, Basel, Switzerland. This article is an open access article distributed under the terms and conditions of the Creative Commons Attribution (CC BY) license (https:// creativecommons.org/licenses/by/ $4.0 /)$.
Klifovet AG, Geyerspergerstr. 27, 80698 Munich, Germany; Sabine.Richter@Klifovet.com (S.R.); Julia.Hunte@Klifovet.com (J.H.)

* Correspondence: Klaus.Hellmann@Klifovet.com

+ Presented at the XXXI National Conference of the Italian Society of Parasitology (SoIPa), Online, 16-19 June 2021.

Abstract: Twenty-one years after Directive 2001/82/EC regulating veterinary medicinal products (VMPs) came into force, a new veterinary regulation will apply from 28 January 2022. Future applicants will have to follow the requirements of Regulation (EU) 2019/6. This regulation defines different products, non-biologicals (equivalent to formerly defined pharmaceuticals), and biological products. Biological VMPs belong either to the new class, non-immunological VMPs or immunologicals, including those known as vaccines. This new regulation also defines novel therapies covering innovative medicinal therapeutic approaches. Antiparasitic products will always be VMPs, and additional requirements for environmental safety and potential risks for the development of resistance are enforced.

Keywords: Regulation (EU) 2019/6; veterinary regulation; veterinary medicinal products; antiparasitics; environmental risk assessment; resistance

\section{Introduction}

Antiparasitics (AP) are substances that kill or interrupt the development of parasites. Within the European Union (EU), APs fall under the definition of VMPs. VMPs are substances or combination of substances that treat or prevent a disease or that restore, correct or modify physiological functions. Antiparasitics are used for the purpose of treating or preventing an infection, infestation or disease caused or transmitted by parasites, including substances with a repelling activity. The legislative framework related to the authorisation and marketing of VMPs in the EU will change in January 2022. The new Regulation (EU) 2019/6 will make a change to the requirements for the authorisation of novel APs. An overview of these changes, their impact and the authorisation process of VMPs in the EU from 28 January 2022 is given in the following sections.

\section{Regulation (EU) 2019/6 on VMPs}

Twenty years after the inception of Directive 2001/82/EC regulating the authorisation of VMPs in the EU, a new legal framework will apply from 28 January 2022-Regulation (EU) 2019/6. Its content stays abreast of changes in the scientific progress, the current market conditions and the economic reality in the EU. This regulation defines different product categories, non-biologicals (equivalent to those formerly defined pharmaceuticals), and biological products. Biological VMPs belong either to the new class, non-immunological VMPs or immunologicals, including those known as vaccines. The new regulation also defines novel therapies covering innovative medicinal therapeutic approaches. Under Directive 2001/82/EC, it was sometimes challenging to assign the appropriate category and legal requirements to new therapies. Novel antiparasitic products will have to follow the requirements for each of the different classes. This includes a highly demanding manufacturing process to guarantee for the quality of the product, the demonstration of the 
safety for the target species, the environment, the user and possibly the consumer. The fate of potential residues in food-producing species needs to be evaluated, and a high level of efficacy of the product is paramount, this being the benefit of its use. There are, however, certain provisions that will apply to antiparasitics in general, having a potential impact on the development and marketing authorisation process of such products.

\section{Environmental Concerns Related to Antiparasitics}

Particularly pharmaceutical VMPs, defined as non-biological products - the majority of antiparasitics belong to this class-may have a relevant impact on the environment. The environmental impact assessment has been part of a marketing authorisation in individual European countries for several decades. In 1993, it became a requirement, as stated in Directive 92/18/EEC [1]. Constantly extended, current guidance documents for the environmental impact assessment were released in the early 2000s. In 2000 and 2005, the Veterinary International Conference on Harmonization (VICH) published the requirements for the two phases of assessment of the risk for the environment: VICH guideline (GL) 6 for Phase I and VICH GL 38 for Phase II. The assessment in Phase I identifies those pharmaceuticals that have a limited effect on the environment. This usually includes VMPs with a finite exposure to the environment, e.g., VMPs used for the treatment of individual animals rather than groups. VMPs exceeding the defined threshold concentrations in soil or water, or exhibiting certain characteristics, are subject to the ecotoxicity assessment of Phase II.

The new regulation puts a stronger focus on the benefit:risk evaluation of authorised VMPs. For antiparasitic products, the assessment of their influence on the environment carries more weight. This reflects the increasing awareness of residues originating from pharmaceuticals in soil and water, including the European Commission's Strategic Approach to Pharmaceuticals in the Environment [2]. Since the publication of VICH GL 38 for the Phase II environmental risk assessment in 2005, 17 pharmaceutical veterinary medicinal products have been re-assessed in view of their potential risk to the environment [1]. Among those products, eight were antiparasitics (including two coccidiostats). The concerns associated with the eight products were their persistence in the soil, the risk they pose for the dung fauna, the inadequacy of the provided data on environmental risks and/or discrepancies in the risk mitigation measures in relation to the risks to the environment.

The reason for one of the re-assessments was denoting for antimicrobials: the substance was assumed to be a potential PBT substance. These compounds fulfil the persistence, bioaccumulation and toxicity criteria determined by the so-called REACH Regulation (EC) 1907/2006, concerning the registration, evaluation, authorisation and restriction of chemicals. PBT, as well as vPvB (very persistent and very bioaccumulative), substances pose a huge threat to the environment, but potentially also to human health. In the future, under the new Regulation (EU) 2019/6, antiparasitics will not be approved in the EU if they are a PBT or a vPvB substance. However, there is one particular exception: if the applicant can prove that the product is essential for the control or prevention of a serious risk to the health of the target animals, the conclusion on the benefit-risk assessment may still be in favour of the antiparasitic.

\section{Development of Resistance and Regulation (EU) 2019/6}

Beyond the increased awareness of environmental effects, the occurrence of antiparasitic resistance is in the focus of the new Regulation (EU) 2019/6. Antiparasitic resistance is the genetic ability of parasites to survive treatment with an antiparasitic drug that was effective against the parasites in the past. The properties of the drug, practices of treatment and livestock management practices, the immune system of the treated animals and the biology of the parasite are all factors that contribute to potential resistance. Nevertheless, it is important to consider that the development of resistance is a natural process in the evolution of parasites. 
To reduce the risk of the development of resistance in parasites, future applications for marketing authorisation will have to address this issue. Data on present or potential future resistance in the target animals will have to be presented. A scientific evaluation of the mechanisms of resistance should be conducted by the applicant. Here, not only the resistance of the antiparasitic is of interest, but also the potential co- and cross-resistance to already authorised products. If the competent authority assessing the marketing authorisation application is of the opinion that the risk to public health by the development of resistance to the antiparasitic is higher than the benefit that comes with the treatment or prevention of parasitic disease, the marketing authorisation will not be granted.

\section{Antiparasitics and Limited Markets}

To encourage the authorisation of VMPs for animal species other than cattle, sheep for meat production, pigs, chicken, dogs and cats (so-called major species) and VMPs intended for diseases occurring infrequently or in limited geographical areas, requirements for limited markets are defined in Article 23 of Regulation (EU) 2019/6. While this article offers the opportunity for easier market access for products intended for limited markets, current proposals by EMA/CVMP exclude antiparasitics from this approach. Due to the high number of different parasites in many different animal species, the authors asked the scientists in veterinary parasitology to start to communicate to the European Commission and EMA/CVMP the importance of easy access to antiparasitic VMPs, including the application of Article 23 for such products.

\section{Conclusions}

The treatment and prevention of parasitic infestations are part of everyday practice in both livestock and companion animals. When, after 21 years, on 28 January 2022 a new regulation will apply, the marketing authorisation requirements for antiparasitics will become more stringent. Regulation (EU) 2019/6 will put a stronger focus on the environmental impact of substances administered to food-producing animals and pets. Antiparasitic resistance, its emergence and prevention will be given more attention when future applications will be evaluated. Although these risks exist, antiparasitics should also benefit from the provisions of Article 23 for limited markets. The new veterinary regulation aims to facilitate the authorisation of new and novel therapies, allowing new technologies to be licensed and up-to-date scientific knowledge to be applied.

Conflicts of Interest: The authors declare no conflict of interest.

\section{References}

1. Fabrega, J.; Carapeto, R. Regulatory review of the environmental risk assessment of veterinary medicinal products in the European Union, with particular focus on the centralised authorisation procedure. Environ. Sci. Eur. 2020, 32, 1-15. [CrossRef]

2. European Commission. European Union Strategic Approach to Pharmaceuticals in the Environment. COM/2019/128 Final. Available online: https:/ / eur-lex.europa.eu/legal-content/EN/ALL/?uri=CELEX:52019DC0128 (accessed on 30 June 2021). 